\title{
A Comprehensive Survey on the Performance Analysis of Underwater Wireless Sensor Networks (UWSN) Routing Protocols
}

\author{
Tariq Mahmood ${ }^{1}$, Faheem Akhtar ${ }^{2}$, Khali ur Rehman ${ }^{4}$, \\ Saqib Ali ${ }^{5}$, Fawaz Mahiuob Mokbal ${ }^{6}$ \\ Faculty of Information Technology, \\ Beijing University of Technology, \\ Beijing, China 100124
}

\author{
Sher Daudpota ${ }^{3}$ \\ Department of Computer Science \\ Sukkur IBA University \\ Sukkur 65200, Pakistan
}

\begin{abstract}
The probe of innovative technologies is a furious issue of the day for the improvement of underwater wireless sensor network devices. The undersea is a remarkable and mystical region which is still unexplored and inaccessible on earth. Interest has been increasing in monitoring the medium of underwater for oceanographic data collection, surveillance application, offshore exploration, disaster prevention, commercial, scientific investigation, attack avoidance, and other military purposes. In underwater milieus, the sensor networks face a dangerous situation due to intrinsic water nature. However, significant challenges in this concern are high power consumption of acoustic modem, high propagation latency in data transmission, and dynamic topology of nodes due to wave movements. Routing protocols working in UWSN has low stability period due to increased data flooding which causes nodes to expire quickly due to unnecessary data forwarding and high energy consumption. The quick energy consumption of nodes originates large coverage holes in the core network. To keep sensor nodes functional in an underwater network, dedicated protocols are needed for routing that maintain the path connectivity. The path connectivity consumes more energy, high route updated cost with a high end to end delay for the retransmission of packets. So, in this paper, we are providing a comprehensive survey of different routing protocols employed in UWSN. The UWSN routing protocols are studied and evaluated related to the network environment and quality measures such as the end to end delay, dynamic network topology, energy consumption and packet delivery ratio. The merits and demerits of each routing protocol are also highlighted.
\end{abstract}

Keywords-Underwater Wireless Sensor Networks (UWSN); routing protocols; end-to-end delay; energy consumptions

\section{INTRODUCTION}

Wireless sensor networks (WSNs) is an emerging technology of the day which is used to structure large number of separated tiny embedded sensor nodes used in monitoring and sensing of data from the aqueous environment [1], [2]. In present years, wireless sensor network applications span in the different fields used in weather monitoring, pollution monitoring, military, health, home, and commercial companies [3].

Wireless sensor networks comprises of structured and unstructured networks for sensing of huge amount of aqueous data [4]. The deployment of structure or unstructured networks is usually depend on the environments that needs to be moni- tored. Mobile Social Networks is a modern distributed buffer storage approach used for data exchange and communication between the mobile users to enhance the network performance concerned to content delivery ratio, e2e delay and throughput [5].

In underwater wireless sensor network; routing is different from the terrestrial wireless sensor networks due to limited bandwidth, energy, node mobility, and end to end delay in the data packet transmission [6]. The energy efficiency is an important prerequisite to their reliable operation and resource management. The routing protocol techniques play an crucial role in energy efficiency that supports network quality of service [7]. Since every application has different quality factors and challenges, there is need to diversify routing protocol having ability to fulfill the application requirements [8].

Fig. 1 illustrate about the architecture of underwater wireless sensor network [9], [10]. In underwater many static acoustic sensor node are distributed over seabed and mobile sensor nodes that move freely with water current. Sensor nodes nous data from environments and detect the movement of submarine that works as autonomous underwater vehicle. In this figure, submarine acquire data from acoustic sensor nodes, aggregate the collected data and store data temporarily. The submarine, static node and mobile sensor node communicate each other and their relevant cluster head through acoustic signals. The submarine forward the aggregated data to surface sink base station. Surface Sink Base station froward the received data to man-controlled computer using radio signal through satellite communication.

TDOA localization (Time difference of arrival technology) is a significant technology instigated in sensor node to ascertain the source location in the real time or time critical applications to evade from interruption arise due to multipath channel [11]. The autonomous underwater vehicle (UUVs, AUVs, Submarine) move in water in fixed pattern to communicate with sensor nodes through short range and high rate data link. AUVs near the base station negotiate to the sink node to forward the collected data. The efficient underwater communication poses significant problems due to intrinsic absorption nature of water. Currently, there has been a growing awareness in monitoring underwater media, analysis of water quality, water pollution monitoring [12], [13] such as biological, chemical 


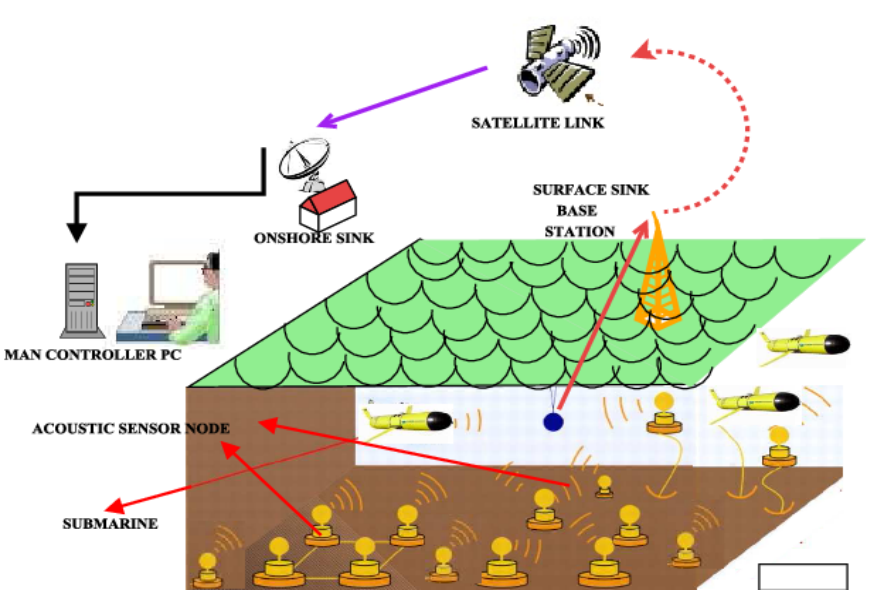

Fig. 1. Underwater Sensor Network Architecture.

and nuclear, micro-organism or fish movement tracking, pressure movements, temperature movement, disaster prevention, underwater oil and gas pipeline corrosion detection, military and home land security application [14], [15]. The acoustic signal also faces many problems due to high error rate, low available bandwidth, node mobility, less propagation speed, a wide class of security threats and malicious attacks and high end to end delay [16], [17]. In recent years the wireless sensor network applications span in the different fields that are used in weather monitoring, water pollution monitoring such as biological, chemical, nuclear, micro-organism tracking, and disaster prevention [18]. In UWSN, link failure is burning issue due to node mobility. Node mobility will create the holes in the network causing increase in end to end delay in data transimission. Existing routing protocols have been analyzed which investigate the optimization performance of network services, node mobility, end to end delay and energy draining of sensors nodes. Large number of routing protocol have been developed working in UWSN [19], [20]. These protocols evaluate the performance efficiency in respect to end to end delay, node mobility, network throughput, and energy consumption. In order to design the efficient UWSN routing protocol researcher faces many challenges which attenuated to the medium are node mobility, end-to-end propagation delay and energy saving [21].

This rest of the paper is organized as follows, Section 2 presents overview and working of UWSN routing protocol. Section 3 presents the comparison of different routing protocol working in underwater wireless sensor network. Section 4 presents the detail of comparative results. Finally, the paper is concluded and future research directions and issues are pointed out in Section 5.

\section{A. Major Challenges in Design of UWSN}

Factors affecting on the propagation of underwater acoustic signals have become the designing challenges for UWSN. Following are the factors that effect on propagation of underwater acoustic signals: [22]

- $\quad$ Bandwidth: The bandwidth available is extremely limited due to water absorption.
- Propagation delay: In underwater network, the propagation delay is five times higher than RF (terrestrial channel). The RF speed is 3x108 ms-1 whereas speed of acoustic signal is about $1.5 \times 103$ ms-1. The low speed of sound causes multi-path propagation to stretch over time delay. It effects real time application of UWSN.

- $\quad$ Shadow zones: Due to the underwater extreme characteristics like density and temperature, high bit error rates and temporary losses of connectivity occur.

Energy: Limited battery power is difficult to recharge.

Attenuation: Due to decreased amplitude and intensity of a signal.

The devices for underwater sensor networks are more expensive and have limited availability in market.

- $\quad$ Noise from machinery, shipping and movement of the fish or animals are concerned in UASN.

\section{B. Differences between UWSN and TWSN}

The underwater wireless sensor network is different from the terrestrial wireless sensor network due to the unique characteristics of the water. Following are the major differences shown UWSN and TWSN [23], [24]

- $\quad$ Communication Method: WSN uses radio signals whereas UWSN uses acoustic signals.

- $\quad$ Cost: Terrestrial sensors are inexpensive while underwater sensors are expensive due to transceivers complexity and protection.

- $\quad$ Power: UWSN needs more power than the TWSN because acoustic signals cover long distance and more complex signal techniques are implemented whereas RF needs less power.

- Memory: UWSN requires more memory for caching the data because the connection of acoustic signal can be disabled by shadow zones (less than 100 meters area) while this issue is not treated in terrestrial WSN.

- Difference in Deployment: The sensors are deploying densely in terrestrial sensor application like in tracking system while it will be costly in UWSN to deploy densely. It is not easy to deploy sensors in UWSN in densely.

- $\quad$ Performance: The performance of TWSN is better than UWSN.

- $\quad$ Mobility: Sensor nodes in underwater are mobile whereas in terrestrial network they are fixed

\section{Routing Protocols}

The routing is the basic task of network layer used to determine the route from source to destination. The network layer is the administrator that tells how the messages are routed within the networks. In Underwater WSN; routing is different from the terrestrial WSN due limited amount of bandwidth, node mobility for ocean current and end to end delay in data packet transmission [25]. Therefore, in order to hold the network together, there is a need to develop the routing strategies. The design of routing protocol for UWSN is 
concerned with saving energy and node mobility in the longterm non-time critical applications. The researchers have made numerous efforts to develop efficient routing protocol while considering the unique characteristics of underwater network [26].

There are mainly three categories namely that determine the path:

1) Proactive Routing Protocols (Table Driven): The core function of this protocol is to maintain the routing table containing all routing information to find routes from node to node [25], [27], [28]. This protocol reduces message latency brought by routing discovery. The proactive routing protocol first generates a signal on predefined route to establish the route. All nodes update route information in their routing table. The protocol establishes the route because every time topology is modified due to link failure and node failure in underwater wireless sensor networks. In UWSN, memory and energy are main reasons to avoid the proactive routing protocol in UWSN.

2) Reactive Routing Protocols: In reactive routing protocol, node start the route finding process when a route is needed to destination. Once route is established, it is maintained by routing table and is remained in routing table until it is needed. This protocol is more suitable for dynamic environments. This protocol is usually used by source initiated by flooding method [25], [27], [28]. This results an increase in message latency unsuitable for UWSNs.

3) Geographic Routing Protocols: Source to destination path is established in geographic protocol by controlling location information[25], [27], [28], [29]. In this scenario, source node selects next forwarder node that is based on the location information of neighbour node. In the underwater environments, it is challenging to attain an accurate location information due to the node movement in water current.

This paper is organized as follows. Section 2 presents overview and working for different routing protocols working in Underwater Wireless sensor network. Section 3 presents evaluations and analysis of different routing protocols based on information of different metric regarding network performance, architecture and design. Section 4 presents the conclusion and point out the future open research issues.

\section{BACKGROUND}

This section describes the functioning of different routing protocol operational in Underwater Wireless Sensor Network. Metric like node mobility, end to end delay, deployment, routing approach, energy consumption and packet delivery ratio are used as depiction of routing protocol. According to our review and explored search material we come to know three major categories of these protocols namely Geography based, flat based/Multipath based and clustering/hierarchical based routing protocols.

\section{A. Geography based Routing Protocol}

Location information is required to determine the distance between two selective nodes so that end to end delay and energy consumption can be calculated approximately. This category is subdivided into following specific routing protocols; these are VBF, HH-VBF and AURP. Let us discuss the each of them in the following subsections.
TABLE I. VBF DATA PACKET [8]

\begin{tabular}{|c|c|c|c|c|c|}
\hline $\begin{array}{c}\text { Features } \\
\text { subset }\end{array}$ & $\begin{array}{c}\text { Source } \\
\text { Position }\end{array}$ & $\begin{array}{c}\text { Target } \\
\text { Position }\end{array}$ & $\begin{array}{c}\text { Forwarder } \\
\text { Position }\end{array}$ & $\begin{array}{c}\text { Range } \\
\text { Field }\end{array}$ & $\begin{array}{c}\text { Radius } \\
\text { Field }\end{array}$ \\
\hline
\end{tabular}

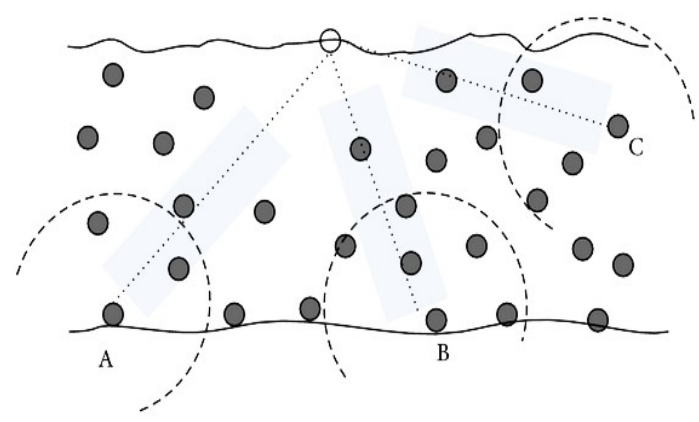

Fig. 2. VBF Routing Protocol with a Single Virtual Path.

1) Vector-Based Forwarding Routing Protocol: VBF is geographically based (location-based) routing protocol, proposed for Underwater WSN [30], [31]. The VBF routing protocol handle node mobility and maintains the routing path at medium level. The VBF gives high data delivery rates, energy efficiency and robust. There is no node state information is required because all nodes are involve in packets forwarding. Data packets is forwarded with redundant path from sender to destination. Sink deals with node failure and packet loss problem.

Routing: Table I illustrates about the all field of data packet routed in VBF routing protocol. Each data packets carries routing information of source position, target position, forwarder position. The Range field is used to handle the node mobility and Radius field which contain pre-defined threshold width that is used to decide as a forwarder by sensor nodes if they are nearby the routing pipe.

Fig. 2 explains, how VBF routing protocol build a virtual path in network and clearly show a virtual path build for nodes $\mathrm{A}, \mathrm{B}$, and $\mathrm{C}$. In $\mathrm{VBF}$, a "routing pipe or virtual pipe or vector" is established between sender node to target node that embeds its own position, sink position, and its position as a rely node in the packet and broadcasts this packet.. All packets are forwarded through this vector pipe from source to sink node. Only node that close to the vector pipe have ability to forward the data packets from source node to destinated sink. Routing pipe have not only limiting the network traffic significantly but also can easily control the dynamic topology. Every node keeps the information of its location. All the nodes estimate their position by determining the forwarder distance and Angle of Arrival (AoA) of receiving packet. If a node is near to vector pipe with respect to pre-defined threshold distance value, node update its own position in FP field of data packet and forward otherwise discard. VBF is working in two modes: Sink initiated Query:It is location depended query. Sink initiate query for interested area by issuing the INTEREST query packets that contains the source location, target location in the sink coordination system. Source initiated Query:It is location independent query. When 
source is interested to send data after setting its location in the source coordination system, it broadcast $D A T A_{R} E A D Y$ packets to all nodes.

Desirable Factor:The node competence to forward packets to another nodes are calculated by desirable factor.

\section{Merits:}

- $\quad$ Achieve robustness against the node failure.

- In dense area, end to end delay is minimum.

- $\quad$ Energy efficient.

\section{Demerits:}

- In VBF node mobility is not handled efficiently.

- Small data delivery in sparse area because some time, few node lie in routing pipe that are responsible for packet forwarding.

- $\quad \mathrm{VBF}$ is very sensitive for pre-define threshold radius because routing performance can affect by threshold radius. Some nodes send data packets again and again from source to sink which drain their energy and increase end to end delay.

- $\quad$ Multiple node are involved in routing act as relay node.

- Due to 3-handshake nature, VBF produce communication overhead which cannot consider the link quality during this operation.

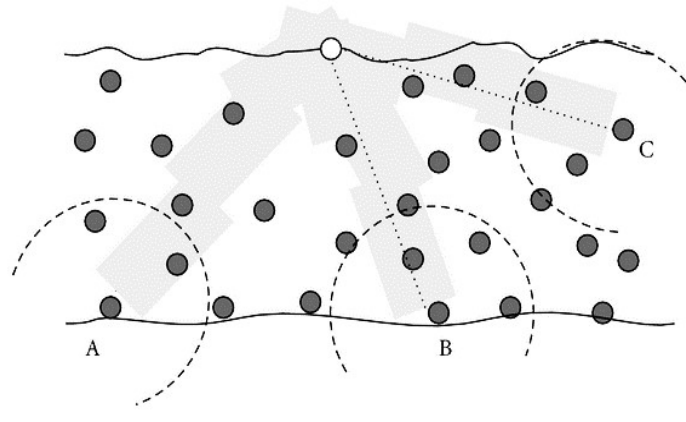

Fig. 3. HH-VBF, Each Node Separate Virtual Path.

2) HH-VBF: Hop by - Hop Vector - Based Forwarding Routing Protocol: Hop-by-Hop Vector Based Forwarding Routing Protocol (HH-VBF) is working similar to VBF routing protocol.

Fig. 3 shows each forwarder node builds its separate virtual pipe instead of one virtual pipe between source and destination node in HH-VBR [32], [33], while in VBF, a single "vector" is built between sender to destination in the entire network.

Routing: In HH-VBF, intermediate node make forwarding decision based on its present location. Forwarding process is similar to VBF, when a node receive a packet, it keeps packet for short time. This awaiting time is proportional to desirable factor that decide which node is suitable as forwarder node. It is calculated by measuring the distance from the routing vector, angle and transmission range between the nodes. On the expiry of awaiting time, node forward that packets which has smallest desirableness factor.

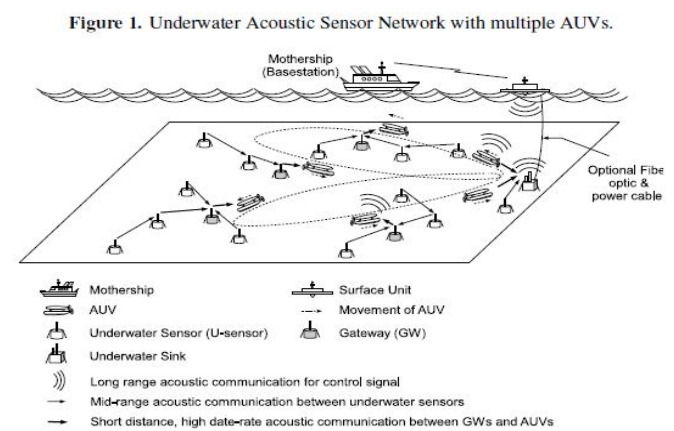

Fig. 4. AUVs working in Underwater Acoustic Network.

Like the VBF, in $\mathrm{HH}-\mathrm{VBF}$ the desirableness factor of each node depends upon:

- Distance of source node to forwarder node.

- Distance of source node to the routing pipe.

- Angle form at forwarder between vector, from forwarder to sink and forwarder to source node.

After recieving a packet, node hold the packet for the time interval $T_{\text {adaptation }}$.

\section{Merits:}

- It gives the better delivery ratio in sparse area.

- Handle node mobility efficiently.

- $\quad \mathrm{HH}-\mathrm{VBF}$ produce the signaling overhearing due to hop by hop nature.

- Ability to identify delivery route information even nodes are scattered in networks.

\section{Demerits:}

- $\quad$ By increasing node density will result to increase end to end delay.

- Node density will result to increase energy consumption.

- $\quad$ Node density will result to low data delivery ratio.

- Handle node mobility at medium level due to hop by hop routing pipe.

- Low energy efficiency.

- Difficult to recharge the battery.

\section{B. AURP: AURP Routing Protocol for UWSN}

AURP Routing Protocol propose to attain the high data rates and low energy consumption in underwater wireless sensor networks [34]. AURP routing protocol use heterogeneous acoustic channels for communication and handle the movement of numbers of AUVs (autonomous underwater vehicles). AUV are used as a relay nodes.

Routing: Fig. 4 explains the network architecture of AURP that comprises of U-Sensor nodes, Gateways, AUVs, and surface node/mother ship. The U-Sensor nodes send the aggregated sense data directly to Gateway by using mid range acoustic channel or by multihop fashion. Gateway forward aggregated data to sink directly or through AUVs when it passes near the Gateway through high data rates channel. 


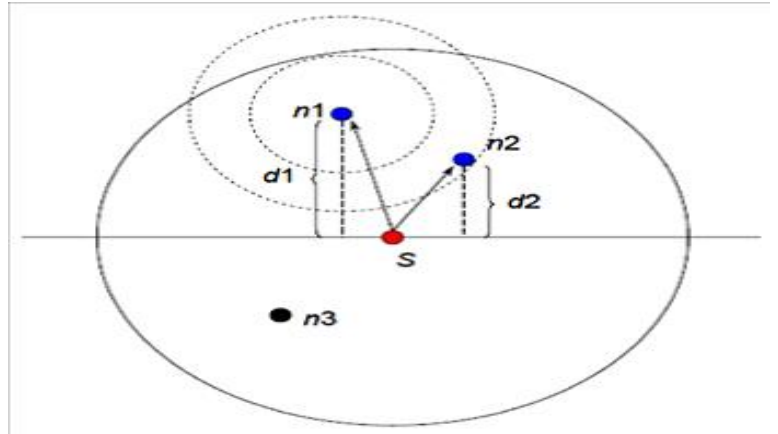

Fig. 5. Selection of Optimal forwarded Node in DBR Protocol.

The AUVs deliver received data to sink using short range high data rates channel. Sink sent aggregated data to surface station through fiber optic cables. The AUVs also use low data rates interface for long distance to sent urgent data. AUVs movement is controlled by surface station by sending control signal. The Gateway and sink broadcast their interest toward the sensor node to receive data periodically. AUVs routing protocol is working in four modes:

Sink Node: A sink node broadcast messages every time which is used by U-Sensor to find the next node. Sink node negotiate with AUVs to establish the link for data transmission. Sink received sense data form the U-Sensor directly or other U-Sensor relaying data.

Sensor Nodes:U-Sensor nodes periodically forward collected data to sink. U-Sensor determine the next node by maintaining the timer.

Gateway Nodes: Gateway node flood messages periodically that is recieved by U-Sensors nodes. Gateway receive data from U-Sensors and stores in its queue until AUVs negotiate with gatewsay. AUVs:Multiple autonomous underwater vehicle collect data from gateway and send to sink.

\section{Merits:}

- $\quad$ Achieve high data rates.

- $\quad$ Low energy consumption.

- $\quad$ Control the mobility of multiple AUVs

Location free routing protocol which need node's related other information like pressure, depth, dynamic address in greedy fashion routing instead of fully geographical node information. Following are the flat based routing protocols in UWSN.

\section{Flat Based / Depth Based Routing Protocol}

1) DBR: Depth-Based Routing : DBR is location based routing protocol use greedy algorithm [35] to forward the packet from source to sink node. Certain protocol required the full dimensional location information of sensor node in underwater wireless sensor network. In DBR routing protocol each node use depth base information. The sensor node equipped with inexpensive depth hardware to calculate the depth pressure locally.

Routing: Fig. 5 shows n1, n2, n3 are neighbor sensor nodes of sensor node $\mathrm{S}$ and circle line represents node $\mathrm{S}$ transmission range. Node $\mathrm{S}$ broadcasts a packet to $\mathrm{n} 1, \mathrm{n} 2, \mathrm{n} 3$ neighboring nodes in transmission rang. Node $\mathrm{n} 1$ and $\mathrm{n} 2$ is qualified forwarding node whereas $\mathrm{n} 1$ is selected as optimal forwarded due low depth and node $\mathrm{n} 3$ is below of $\mathrm{S}$ node, so it discard the packet. Fig. 6 show, multiple stationery data sink are deployed at sea surface in underwater. Sensor nodes are randomly deployed at different depths which sense data from environment and sends data packet containing depth information to its optimal neighboring node through multi-hop fashion (greedy algorithms). On receiving data packets, the neighboring node calculates it depth via pressure hardware and compares it depth "dc" with the packet containing depth "dp". If ( $\left.d c_{j} d p\right)$ node depth is less then packet containing depth, then packets are forwarded to the next node otherwise packets discard. Data packet is deliver by hop to hop manner to surface sink and base station.

TABLE II. DBR DATA PACKET [25]

\begin{tabular}{|l|l|l|l|}
\hline Source ID & Packet Sequence number & Depth & Data \\
\hline
\end{tabular}

In Table II, Data packet of DBR contains source ID, Packet seq \#, depth information and original data. "Source ID" is source node identifier, "Packet sequence" unique sequence \# assign to data packet by the source node, "Depth" is the depth information that is used to update node by node when the data packet is forwarded.

In DBR, there are two factor involve in collisions and

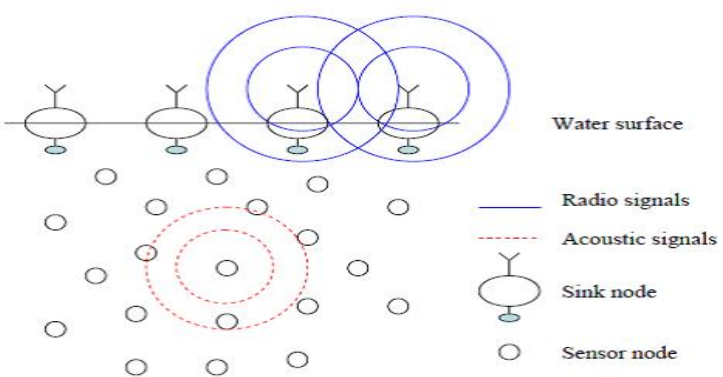

Fig. 6. DBR node deployment and network working architecture.

redundant transmission of packet. First, multiple path forward data from each nodes by flooding approach and second is, every node send the same data packet many time / repeatedly. In order to control the same packet delivery, the DBR uses priority queue to decrease number of forwarded nodes and packet history buffer to handle the packet retransmission.

To prevent from collisions, redundant packet transmission and high overhead, the DBR use holding time for each received packet based on packet embedded depth dp and node own depth dc. Different node have different holding time. Each node wait for holding time when it is expired, it forward the data packet.

\section{Merits:}

- It can easily handle the mobility of nodes and also handle the traffic at multi-sink on ocean surface.

- It achieves high data rates in sparse area.

- $\quad$ No need for full geographical information. 


\section{Demerits:}

- $\quad$ High energy consumption.

- $\quad$ High end to end delay in dense medium.

- Communication problems in sparse area due to greedy algorithms.

- $\quad$ The data packet is forwarded in broadcast fashion so number of duplicate packets are forwarded which decrease the network performance.

- In sparse area, if depth position of two nodes are same, the network performance is reduced because continuously finding the suitable forwarder.

- $\quad$ Due to sparse and dense area, the complexity is also increased which lead not only to consume more energy and packet losses but also in-efficient use of memory.

- $\quad$ Link failure and hole arise due to draining of energy on the top layer that effect on the network performance.

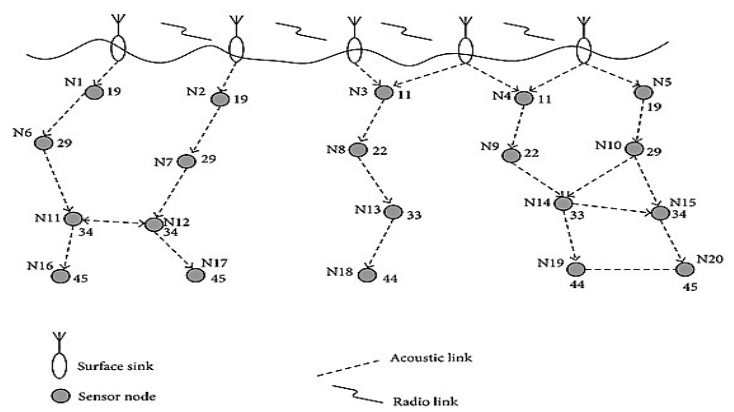

Fig. 7. H2-DAB Hop-ID Assigning Process.

2) Hop-by-Hop Dynamic Addressing Based Routing Protocol: H2-DAB: $H^{2} \mathrm{DAB}$ "Hop-by-Hop Dynamic Addressing Based Routing" is the first greedy and dynamic address based routing protocol in UWSN [36]. The H2-DAB does not require the extra hardware and location information like other greedy protocol in UWSN. The basic purpose of $\mathrm{H} 2-\mathrm{DAB}$ is to solve continuous nodes movement problems. The movement problems is solved by allocating dynamic address to sensor nodes so that the float sensor nodes get the new dynamic address according to their position in different depth level.

Routing: Fig. 7 elaborates the ID assigning process in H2DAB network that consists of multiple static sink located on ocean surface which collect data from sensor fixed with bottom in greedy fashion. The ocean is divided into different depth level (8 to 10 layer) and nodes are deployed randomly move horizontally and vertically. At initial "99", is default Hop ID assigned to every floating node, after receiving Hello packet from sink the node will updated their Hop ID.

$\mathrm{H} 2-\mathrm{DAB}$ is comprising on two different phases 1) assign the dynamically address to static surface nodes, mobile nodes and sensor nodes, 2) sent data by using these addresses.

Table III illustrate about the all field of data packet routed in H2-DAB routing protocolDuring first phase, the dynamic address is allocated to each node by hello message. One static Hop ID is allocated to anchor bottom sensor nodes while the moving node and surface sink are assigned with two type of Hop ID.

TABLE III. DBR Data Packet [25]

\begin{tabular}{|l|l|l|l|l|}
\hline Sender Hop ID & Next Node ID & Packet Seq \# & Destination ID & Data \\
\hline
\end{tabular}

During the second phase, data packets are send toward the sink node. To select the forwarder, source node send a inquiry request message to each neighbor node within its communication range. The less Hop ID range node is selected as forwarder, so data is forwarded toward the sink through greedy fashion. Due to mobility of nodes the Hop ID is updated after a time of interval.

\section{Merits:}

- $\quad$ Gives high data delivery ratio.

- Handle the node mobility without updating the routing table.

- Reduce the congestion of nodes that near to the surface sink.

- It works without extra hardware and maintain any information in routing table.

\section{Demerits:}

- It is difficult to deploy the mobile nodes at different layer as compared to random deployment.

- $\quad$ Nodes near to sink are working frequently causing drain large amount of energy.

- Communication problem in sparse area due to greedy algorithms is not consider.

- $\quad$ Dynamic addressing phase is completed in short period of time which decreases the network performance.

- $\quad$ Link quality is not considered by single hop that will result in high packet loss and reduce the reliability of network.

3) Energy-efficient depth-based routing protocol for underwater wireless sensor networks: EEDBR is an energy efficient localization free routing protocol working in Underwater WSN. The EEDBR [37] is sender based routing approach in which sender node opt the forwarder node depending on node residual energy and depth information from its neighbor nodes. It does not consider the link failure which is important parameter in ocean environment. EEDBR, void critical problem in greedy approach can't handle. Energy draining in dense network is very high due the unnecessary data forwarding. The low-depth nodes expire earlier due to the increased load of data forwarding, causing a less number of available neighbors for the remaining nodes.

\section{Merits:}

- $\quad$ EEDBR handle the node mobility with water current.

- $\quad$ EEDBR handle the rapid energy consumption of the node near to sink.

- $\quad$ EEDBR is greedy routing approach that uses only depth information and residual energy. There is no need of full location information about the sensor node for routing. 


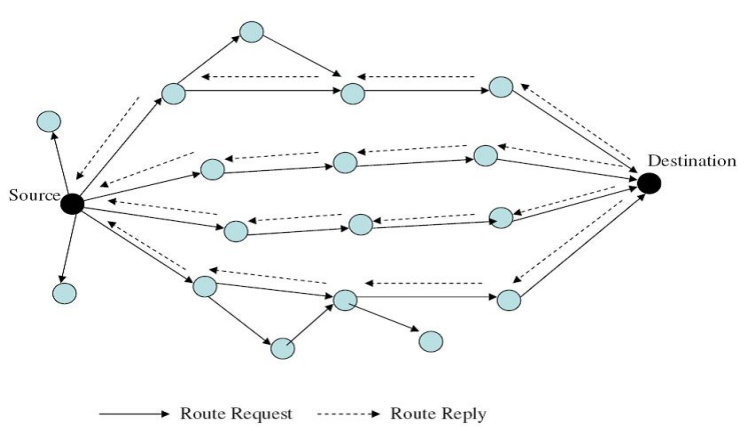

Fig. 8. MPR Basic Procedure.

\section{Demerits:}

- It does not consider the link failure which is important parameter in ocean environment.

- $\quad$ EEDBR, void critical problem in greedy approach can't handle.

- $\quad$ Energy draining in dense network is very high due the unnecessary data forwarding.

- $\quad$ The low-depth nodes expire earlier due to the increased load of data forwarding, causing a less number of available neighbors for the remaining nodes.

4) Towards Delay-Sensitive Routing in Underwater Wireless Sensor Networks: Delay Sensitive DBR [38] is location free routing protocol working in underwater wireless sensor network that is formulated specially for delay sensitive application. Delay Sensitive DBR is enhanced form of DBR in which routing is carry out depend on hold time and depth information of the node. All ordinary sensor nodes forward the sensed data within their transmission scope. A neighbor node that is located in low depth area from the source node calculates their holding time to receive packet. DSDBR working as a greedy algorithm in which data packet is forwarded from source node toward base station through multi-hop fashion. Each qualified neighbor node calculates the forwarding value for the received data packet that is helpful to compute the holding time.

$$
H_{t}=\frac{\left(\alpha-(T L)_{i} q_{i} / \mu\right) H_{-} T_{\max }}{v_{A C}\left(T L \_\min \right)}
$$

Where $H_{t}$ is holding time of received data packet that calculates by each node during which a node stay data packet in its buffer. TL is received packet transmission loss that measure in $\mathrm{dB} . \mathrm{q}$ is the speed in $\mathrm{m} / \mathrm{s}$ of received packet. $\mu$ and $\alpha$ is constant depend on network scope. $H \_T_{\max }$ is maximum holding time of received packet express in sec. $v_{A C}$ is acoustic signal speed denoted in $\mathrm{m} / \mathrm{sec}$. $T \_$min is the minimum transmission loss between two sensor node that is express in $\mathrm{dB}$. An optimal value of holding time is used by sensor node to limiting the redundant packet transmission. The low depth nodes will not forward the packet on overhearing the received packet. Therefore the DSDBR result the minimize the end to end delay by using of holding time and weight function. In stability period of network, there is trade of among throughput and end to end delay.

\section{Merits:}

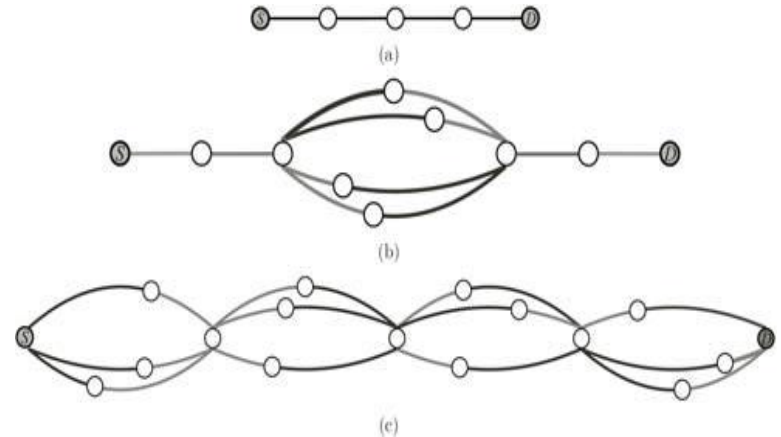

Fig. 9. (a) Single Path, (b) Partial Multipath, and (c) Multipath.

- Networks try to eradicate the distance transmission by the selection of optimal forwarder node depending on holding time and received packet transmission loss.

- DSDBR exhibit a reduced amount of end to end delay by compromising on low stability period and lesser throughput.

\section{Demerits:}

- $\quad$ The constant depth threshold causes the selection of the same nodes as data forwarders again and again; resulting the quick energy consumption of these nodes.

- It faces tradeoff between minimize the end to end delay and increase in consumption of total energy. - It show low network stability period that decrease the network performance.

\section{MPR: Multipath Routing}

The MPR [39] Routing Protocol construct a routing path form source to destination node. During construction of routing path, multipath is utilized between the sender and receiver node which contains a series of Multi-subpath. Multipaths is a subpath from source to it two-hop neighbor node via a relay node in the neighbourhood of both source and destination nodes.

Routing: Fig. 8 shows the construction of routing path, multipath is utilized between the sender and receiver node. Multipaths is route of two-hop neighbor node via a relay node in the neighbourhood of both source and destination nodes. Nodes anchor at sea bottom forward sense data toward the sink at ocean surface. These data packets divided into time slot by source node based on bandwidth. Two hop transmissions are used to send data packets. The destination node receives many packets from different relay nodes, so the MPR prevents from collision at receiving node. The packet arrival is different with different multisubpath.

Fig. 9 (a). There is a single path for data flow between two nodes. The single path waste time during data transmission because it is not fault tolerant. Fig. 9 (b and c), There are multiple path for data transmission between two nodes which have load balancing advantage. In multiple path load balance is attained that decrease the packets drop ratio. The high robustness is also achieved in multipath. Battery lifetime is 


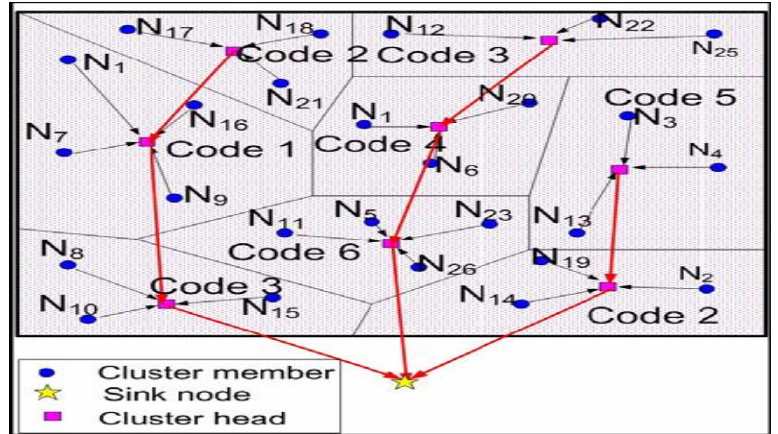

Fig. 10. DUCS, Node deployment and working Network.

improved in multipath routing protocol.

The MPR complete their operation into three phases:

- $\quad$ In first phase, the sender establish routing path. The sender node is required to keep the information of two hop relay from its nearest neighbor node and send to the next hop.

- In second round, the intermediate node "I" is selected by using these information which is collected from propagation delay.

- In third phase, source node check each node to avoid from collision.

\section{Merits:}

- It has higher throughput in dense area while in sparse area that has low throughput.

- It uses multiple paths and therefore has more overhead.

- It has low end to end propagation delay due to multiple path available.

- $\quad$ It has high packet delivery ratio.

\section{Demerits:}

- It deals high energy consumption.

- It uses wire for connection.

- $\quad$ Redundant node create the backup route.

- Redundancy creates the contention among nodes.

\section{E. ARP: Adaptive Routing Protocol}

Adaptive Routing Protocol [40] is used in underwater wireless sensor networks which perform adaptive routing based on application requirements and messages nature. Due to node movement and sparse deployment, UWSN is divided into layers. To achieve the routing performance requirements, protocol get the message redundancy and resource rearrangements. To control packet adaptively, various type of messages have different requirements. The aim of adaptive protocol is to attain trade-off between delivery ratio, energy consumption and propagation delay. The Adaptive routing protocol also provide different services based on data priority level.

Routing: Underwater which float freely in two Dimensional plane controlled by the buoyancy nodes. Sink node is placed

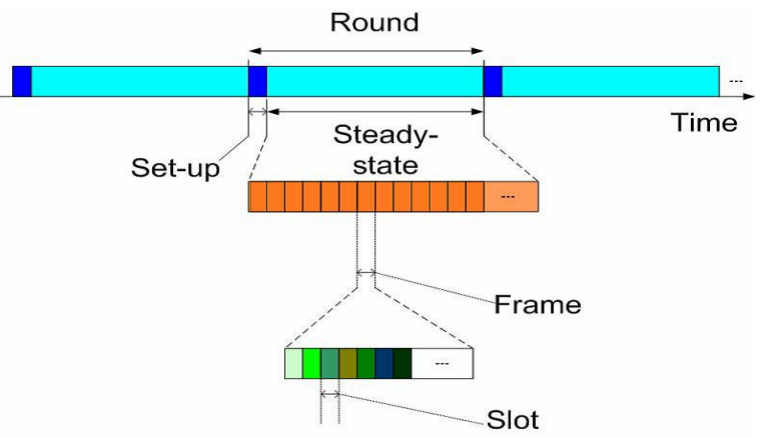

Fig. 11. DUCS Time Line for Node Selection.

in center of water surface. All sensor nodes have knowledge about their position due to the localization algorithms.

Sensor nodes use two types of packet, HELLO packet and data packet. HELLO packet contains information exchange with the neighbor nodes. HELLO packet also contain neighbor discovery information. The data packet is sited in payload. Each sensor nodes perform three types of action, 1) Discovery of neighbor; 2) Calculate priority; 3) Decision for routing. In neighbor discovery mode, each node broadcast HELLO packet to other nodes periodically. The piggy-back ACKs approach is used by each node to broadcast HELLO packet.In second step, packet priority is calculated by vector information which contains, packet age, emergency level, nodes density and battery status. In last step, routing decision is divided into four level. Each level is corresponding to a routing state.

\section{Merits:}

- $\quad$ Achieve different set of services for different types of data packets based on priority.

- $\quad$ It achieves high delivery ratio.

- It is reliable and also efficient for its bandwidth and energy.

\section{Demerits:}

- $\quad$ Performance is not good in UWSN.

- $\quad$ Trade-off among energy consumption and delivery ratio.

- $\quad$ Node Mobility is not handled.

- It is not able to analyze the network performance.

\section{Clustering Based / Hierarchical Routing PROTOCOL}

In hierarchical routing Protocols, nodes are arranged into clusters where a node having low energy can be used to sense the data from its surrounding and forward the sensed data to respective cluster head while a node having high energy can be elected as a cluster head to aggregate the data received from the sensor node and throw it to sink [41]. By this not only the reduction of energy consumption,but it also achieves the equalization of traffic load and scalability. In this scheme following routing protocols are used along their overview, merits and demerits.

Routing: Fig. 10 explains the sensor nodes are structured into cluster where one sensor node is selected as a cluster head for 
TABLE IV. COMPARISON OF UWSN ROUTING PROTOCOLS

\begin{tabular}{|c|c|c|c|c|c|c|c|c|}
\hline $\begin{array}{l}\text { Protocol } \\
\text { Protocol }\end{array}$ & $\begin{array}{l}\text { Routing Approach } \\
\text { Routing Approach }\end{array}$ & $\begin{array}{l}\text { Deployment } \\
\text { Deployment }\end{array}$ & $\begin{array}{l}\text { End-2-End } \\
\text { Delay }\end{array}$ & $\begin{array}{l}\text { Mobility } \\
\text { Mobility }\end{array}$ & $\begin{array}{c}\text { Energy } \\
\text { Efficiency }\end{array}$ & $\begin{array}{c}\text { Delivery } \\
\text { Ratio }\end{array}$ & $\begin{array}{c}\text { Localization } \\
\text { needed }\end{array}$ & Rate \\
\hline $\begin{array}{c}\text { VBF } \\
{[8,9,10,17]} \\
\end{array}$ & $\begin{array}{c}\text { Geography base } \\
\text { (Flooding) }\end{array}$ & Dense & Low & Low & Medium & Low & Yes & $\begin{array}{l}\text { two packet per second } \\
\text { Packet size: } 76 \text { byte }\end{array}$ \\
\hline $\begin{array}{c}\text { HH-VBF } \\
{[9,10,17]}\end{array}$ & $\begin{array}{l}\text { Geography base } \\
\text { (Flooding) }\end{array}$ & $\begin{array}{c}\text { Dense \& } \\
\text { Sparse }\end{array}$ & High & Medium & Low & Medium & Yes & $\begin{array}{l}\text { one packet per } 10 \text { second } \\
\text { Packet size: } 50 \text { byte }\end{array}$ \\
\hline $\begin{array}{c}\text { AURP } \\
{[16]}\end{array}$ & $\begin{array}{l}\text { Geography base } \\
\text { (Flooding) }\end{array}$ & $\begin{array}{c}\text { Dense \& } \\
\text { Sparse }\end{array}$ & Medium & Low & Medium & High & Yes & $\begin{array}{c}48 \\
\text { kbps }\end{array}$ \\
\hline $\begin{array}{c}\text { DBR } \\
{[10,12]}\end{array}$ & $\begin{array}{c}\text { Flat base/depth } \\
\text { (Flooding) }\end{array}$ & $\begin{array}{c}\text { Dense \& } \\
\text { Sparse }\end{array}$ & High & Medium & Low & High & Partially & $\begin{array}{l}\text { one packet per second } \\
\text { Packet size: } 50 \text { byte }\end{array}$ \\
\hline $\begin{array}{c}H^{2} \text {-DAB } \\
{[13,17]} \\
\end{array}$ & $\begin{array}{l}\text { Flat/Addresss base } \\
\text { (Flooding) }\end{array}$ & Dense \& & Medium & Medium & Medium & High & No & $\begin{array}{l}\text { one packet per second } \\
\text { Packet size: } 50 \text { byte }\end{array}$ \\
\hline $\begin{array}{c}\text { MPR } \\
{[15]} \\
\end{array}$ & $\begin{array}{l}\text { Flat base } \\
\text { Multipath }\end{array}$ & Dense & Medium & Low & Medium & Medium & Yes & $\begin{array}{c}10 \\
\text { kbps }\end{array}$ \\
\hline $\begin{array}{c}\text { ADOPTIVE } \\
\text { [18] }\end{array}$ & Flat/Priority base & $\begin{array}{c}\text { Dense \& } \\
\text { Sparse }\end{array}$ & Medium & Low & Medium & High & Yes & $\begin{array}{c}\text { one packet per second } \\
\text { (speed } 0-5 \mathrm{~ms} \text { ) }\end{array}$ \\
\hline $\begin{array}{c}\text { DUCS } \\
{[14]}\end{array}$ & $\begin{array}{c}\text { Clustering } \\
\text { (Distributed) }\end{array}$ & Dense & High & Medium & Medium & Medium & No & $\begin{array}{c}6.6 \mathrm{kbps} \\
\text { (speed } 1.5 \mathrm{~ms} \text { ) }\end{array}$ \\
\hline $\begin{array}{c}\mathrm{MCCP} \\
{[14]} \\
\end{array}$ & $\begin{array}{c}\text { Clustering } \\
\text { (Distributed) }\end{array}$ & $\begin{array}{c}\text { Dense \& } \\
\text { Sparse }\end{array}$ & High & Medium & High & Low & Yes & $\begin{array}{l}2 \text { to } 3 \mathrm{~m} / \mathrm{sec} \\
(3-5 \mathrm{~km} / \mathrm{h})\end{array}$ \\
\hline $\begin{array}{c}\text { EEDBR } \\
{[23]}\end{array}$ & $\begin{array}{c}\text { Depth } \\
\text { (Flooding) }\end{array}$ & $\begin{array}{c}\text { Dense \& } \\
\text { Sparse }\end{array}$ & Medium & High & Low & Medium & Partially & $\begin{array}{c}64 \text { bytes } \\
\text { every } 15 \text { seconds }\end{array}$ \\
\hline $\begin{array}{c}\text { DSDBR } \\
{[22]}\end{array}$ & $\begin{array}{c}\text { Depth } \\
\text { (Flooding) }\end{array}$ & $\begin{array}{c}\text { Dense \& } \\
\text { Sparse }\end{array}$ & Low & Low & Low & High & No & $\begin{array}{c}10 \text { bytes } \\
\text { (speed } 2-3 \text { knots) }\end{array}$ \\
\hline
\end{tabular}

all other nodes. All cluster member nodes forward data packets to their respective cluster head. The cluster head is used for aggregation process on sensed data.Aggregated data is sent to sink through multihop routing. The cluster head performed aggregation function on data and send aggregated data to sink through multihop routing. The cluster head is responsible for inter-cluster and intra-cluster communication. The cluster head randomly selected in order void the draining of battery.

Fig. 11 explains the DUCS routing protocol operation is divided into two rounds, setup round and steady-state round. In steady state, a network is distributed in cluster and data frame is formed with unique id. In steady state rounds, sensor node send numerous frames to each cluster head according to their schedule that composite of series of data massage.

Merits:

- It achieves high data delivery ratio.

- It has lower network overhead and increase the network throughput.

- It is energy efficient.

\section{Demerits:}

- $\quad$ The node mobility affect on cluster structure and reduce cluster life.

- $\quad$ A cluster head only forward the aggregated data to another cluster head.

- $\quad$ Due to water current, the cluster head move away from each other. So they cannot communicate with each other, if any other cluster is not laid down between them.

1) MCCP: Minimum Cost Clustering Routing Protocol: In terrestrial wireless networks, LEACH protocol is proposed in which cluster are made with optimal number of cluster head using distributed approach. The cluster head have constant knowledge about node distribution. Due to water intrinsic nature and non uniformly deployment of nodes in underwater, LEACH is unsuitable in underwater sensor network. The cluster head is made without assuming the constant node distribution. The cluster head movement is also not considered therefore traffic load in different area is un-balanced. Both factors are based on centric approach of cluster head. The MCCP protocol [42] is recommended to handle these problem and increase energy proficiency and prolong network life.

Routing:MCCP exploit cluster base methodology in which the cluster is formed by using the three parameters.

1). Total energy needed to send data form cluster member to cluster head; 2) remaining energy of cluster members and its cluster head; 3) position of cluster head and its relative members. MCCA (minimum cost clustering algorithms) use centralized methodology to choice the cluster head. The MCCP is advanced of MCCA, in which distributed approach is used to select the cluster head.

By this approach, all sensor nodes are candidates for the cluster members as well as cluster head. Neighbor sets and non neighbor sets are constructed by each candidate in order to form the cluster head. Particular cluster head calculates its average cost and broadcast to all members associated with cluster head ID within two hop range. Every cluster member nodes compare their costs with receiving cluster head cost. Cluster member become cluster head, if its cost is minimum than the cluster head cost. It INVITE a message to all other nodes in the cluster to become its members otherwise join message is send to specific cluster head.

Finally a TDMA schedule is defined by the nominated cluster and forwarded to members of the cluster.

\section{Merits:}

- It helps in traffic balancing due to formation of more cluster head.

- $\quad$ Number of Cluster members are depending on the sink location and cluster head. The cluster near to sink has less quantity of cluster member.

- $\quad$ It is capable for load traffic balancing by reclustering node periodically. 
Demerits:

- It does not support in multi-hop fashion.

- Re-clustering is completed in many days or months.

- $\quad$ Due to mobility, different nodes leave and enter the cluster can reduce the cluster efficiency.

\section{COMPaRison OF UWSN Routing PROTOCOLS}

This section describes a comparison in the form of table for different routing protocol functioning in Underwater Wireless Sensor Network. Metric like node mobility, end to end delay, deployment, routing approach, energy consumption and packet delivery ratio are used for analysis of these routing protocols.

In Table IV, routing methodology carries about networks performance enhancement in UWSNs, however it may also faces a variety of intrinsic challenges challenges such as node high mobility due to water flow, 3D environment, high path loss, high rout update cost, low bandwidth, and high propagation delay. Today, there is no any routing protocol can knob all of these challenges. This paper highlighted the basic issues of acoustic communications, data routing and difference between the TWSNs and UWSNs. A collection of such challenges of UWSNs and comprehensive analysis on routing protocols of Underwater Wireless Sensor Networks are briefly discussed as shown in Table IV.

In accordance with the statistics prerequisite for ascertaining the optimistic progress area toward base station, we distributed routing protocols in three categories. A lot number of wellknown routing protocols were studied and their merit and demerit are explained with respect to categories. Additionally, pros and cons of each discussed routing protocol is presented which may critically evaluate functionality of each discussed protocol. Furthermore, these protocols were compared to each other based on their features and their simulation conditions. As per literature studied, we can presume the following assumptions as motivating factors which is essential to publish a survey that mainly focuses on those protocols performing issues \& challenges on routing / channel assignment at network layer.

\section{CONCLUSion AND Future Work}

Routing is an underlying issue of any network, especially in the underwater wireless sensor network. Routing protocols are used to find out different routes that a packet should track over a topology. The design of efficient routing protocol is the fundamental and critical issue of a network layer. The presented research highlights the node mobility and end to end delay issues of UWSN routing protocol at the network layer. The primary motivation of this research is to investigate the performance of different UWSN routing protocols considering different scenarios which are in high demand these days. The researchers made numerous efforts to develop an efficient routing protocol while recognising the unique characteristics of an underwater network. In future work, it is highly needed to create such a routing strategies which can be used to hold the network together i-e underwater and land network, the saving of energy, end to end delay, and node mobility.

\section{REFERENCES}

[1] G. Han, J. Jiang, C. Zhang, T. Q. Duong, M. Guizani, and G. K. Karagiannidis, "A survey on mobile anchor node assisted localization in wireless sensor networks." IEEE Communications Surveys and Tutorials, vol. 18, no. 3, pp. 2220-2243, 2016.

[2] I. F. Akyildiz, D. Pompili, and T. Melodia, "Underwater acoustic sensor networks: research challenges," Ad hoc networks, vol. 3, no. 3, pp. 257279, 2005.

[3] G. Xu, W. Shen, and X. Wang, "Applications of wireless sensor networks in marine environment monitoring: A survey," Sensors, vol. 14, no. 9, pp. 16932-16954, 2014.

[4] J. Yick, B. Mukherjee, and D. Ghosal, "Wireless sensor network survey," Computer networks, vol. 52, no. 12, pp. 2292-2330, 2008.

[5] R. Akhtar, S. Leng, I. Memon, M. Ali, and L. Zhang, "Architecture of hybrid mobile social networks for efficient content delivery," Wireless Personal Communications, vol. 80, no. 1, pp. 85-96, 2015.

[6] U. Prathap, P. D. Shenoy, K. Venugopal, and L. Patnaik, "Wireless sensor networks applications and routing protocols: survey and research challenges," in Cloud and Services Computing (ISCOS), 2012 International Symposium on. IEEE, 2012, pp. 49-56.

[7] K. Song, B. Ji, and C. Li, "Resource allocation for relay-aided underwater acoustic sensor networks with energy harvesting," Physical Communication, vol. 33, pp. 241-248, 2019.

[8] A. Yalcuk and S. Postalcioglu, "Evaluation of pool water quality of trout farms by fuzzy logic: monitoring of pool water quality for trout farms," International Journal of Environmental Science and Technology, vol. 12, no. 5, pp. 1503-1514, 2015.

[9] N.-S. N. Ismail, L. A. Hussein, and S. H. Ariffin, "Analyzing the performance of acoustic channel in underwater wireless sensor network (uwsn)," in Mathematical/Analytical Modelling and Computer Simulation (AMS), 2010 Fourth Asia International Conference on. IEEE, 2010, pp. 550-555.

[10] F. Akhtar, J. Li, M. Azeem, S. Chen, H. Pan, Q. Wang, and J.-J. Yang, "Effective large for gestational age prediction using machine learning techniques with monitoring biochemical indicators," The Journal of Supercomputing, online 2019. [Online]. Available: https://doi.org/10.1007/s11227-018-02738-w

[11] I. Memon, D. Jamro, F. A. Mangi, M. Basit, and M. Memon, "Source localization wireless sensor network using time difference of arrivals (tdoa)," International Journal of Scientific and Engineering Research, vol. 4, no. 7, pp. 1046-1054, 2013.

[12] A. Khan and L. Jenkins, "Undersea wireless sensor network for ocean pollution prevention," in Communication Systems Software and Middleware and Workshops, 2008. COMSWARE 2008. 3rd International Conference on. IEEE, 2008, pp. 2-8.

[13] A. Watt, M. R. Phillips, C.-A. Campbell, I. Wells, and S. Hole, "Wireless sensor networks for monitoring underwater sediment transport," Science of The Total Environment, 2019.

[14] H. Saeed, S. Ali, S. Rashid, S. Qaisar, and E. Felemban, "Reliable monitoring of oil and gas pipelines using wireless sensor network (wsn) remong," in System of Systems Engineering (SOSE), 2014 9th International Conference on. IEEE, 2014, pp. 230-235.

[15] F. Akhtar, j. Li, Y. Pei, and M. Azeem, "A semi-supervised technique for lga prognosis," Proceedings of The International Workshop on Future Technology FUTECH 2019, pp. 36-37, 2018.

[16] A. Davis and H. Chang, "Underwater wireless sensor networks," in Proceedings of IEEE Oceans, 2012, pp. 1-5.

[17] G. Yang, L. Dai, G. Si, S. Wang, and S. Wang, "Challenges and security issues in underwater wireless sensor networks," Procedia Computer Science, vol. 147, pp. 210-216, 2019.

[18] E. Felemban, F. K. Shaikh, U. M. Qureshi, A. A. Sheikh, and S. B. Qaisar, "Underwater sensor network applications: A comprehensive survey," International Journal of Distributed Sensor Networks, vol. 11, no. 11, p. 896832, 2015.

[19] J. Yifeng and S. Lin, "Nir: Uwsn routing protocol based on node neighbor information," in Future Information Technology and Management Engineering (FITME), 2010 International Conference on, vol. 2. IEEE, 2010, pp. 219-222. 
[20] A. Celik, N. Saeed, B. Shihada, T. Y. Al-Naffouri, and M.-S. Alouini, "End-to-end performance analysis of underwater optical wireless relaying and routing techniques under location uncertainty," arXiv preprint arXiv:1901.09357, 2019

[21] A. Nayyar, V. Puri, and D.-N. Le, "Comprehensive analysis of routing protocols surrounding underwater sensor networks (uwsns)," in Data Management, Analytics and Innovation. Springer, 2019, pp. 435-450.

[22] D. B. Kilfoyle and A. B. Baggeroer, "The state of the art in underwater acoustic telemetry," IEEE Journal of oceanic engineering, vol. 25, no. 1, pp. $4-27,2000$.

[23] M. T. Kheirabadi and M. M. Mohamad, "Greedy routing in underwater acoustic sensor networks: a survey," International Journal of Distributed Sensor Networks, vol. 9, no. 7, p. 701834, 2013.

[24] M. Ayaz, I. Baig, A. Abdullah, and I. Faye, "A survey on routing techniques in underwater wireless sensor networks," Journal of Network and Computer Applications, vol. 34, no. 6, pp. 1908-1927, 2011.

[25] A. Wahid and K. Dongkyun, "Analyzing routing protocols for underwater wireless sensor networks," International Journal of Communication Networks and Information Security (IJCNIS), vol. 2, no. 3, 2010.

[26] P. Carroll, S. Zhou, K. Mahmood, H. Zhou, X. Xu, and J.-H. Cui, "Ondemand asynchronous localization for underwater sensor networks," in Oceans, 2012. IEEE, 2012, pp. 1-4.

[27] G. G. Xie and J. H. Gibson, "A network layer protocol for uans to address propagation delay induced performance limitations," in OCEANS, 2001. MTS/IEEE Conference and Exhibition, vol. 4. IEEE, 2001, pp. 2087-2094.

[28] S. Iyer and D. V. Rao, "Genetic algorithm based optimization technique for underwater sensor network positioning and deployment," in Underwater Technology (UT), 2015 IEEE. IEEE, 2015, pp. 1-6.

[29] I. Ullah, J. Chen, X. Su, C. Esposito, and C. Choi, "Localization and detection of targets in underwater wireless sensor using distance and angle based algorithms," IEEE Access, vol. 7, pp. 45 693-45 704, 2019.

[30] P. Xie, J.-H. Cui, and L. Lao, "Vbf: vector-based forwarding protocol for underwater sensor networks," in International conference on research in networking. Springer, 2006, pp. 1216-1221.

[31] Y. Bayrakdar, N. Meratnia, and A. Kantarci, "A comparative view of routing protocols for underwater wireless sensor networks," in OCEANS, 2011 IEEE-Spain. IEEE, 2011, pp. 1-5.
[32] H. Bhambri and A. Swaroop, "Underwater sensor network: Architectures, challenges and applications," in Computing for Sustainable Global Development (INDIACom), 2014 International Conference on. IEEE, 2014, pp. 915-920.

[33] N. Nicolaou, A. See, P. Xie, J.-H. Cui, and D. Maggiorini, "Improving the robustness of location-based routing for underwater sensor networks," in Oceans 2007-Europe. IEEE, 2007, pp. 1-6.

[34] S. Yoon, A. K. Azad, H. Oh, and S. Kim, "Aurp: An auv-aided underwater routing protocol for underwater acoustic sensor networks," Sensors, vol. 12, no. 2, pp. 1827-1845, 2012.

[35] H. Yan, Z. J. Shi, and J.-H. Cui, "Dbr: depth-based routing for underwater sensor networks," in International conference on research in networking. Springer, 2008, pp. 72-86.

[36] M. Ayaz and A. Abdullah, "Hop-by-hop dynamic addressing based (h2dab) routing protocol for underwater wireless sensor networks," in 2009 international conference on information and multimedia technology. IEEE, 2009, pp. 436-441.

[37] A. Wahid, S. Lee, H.-J. Jeong, and D. Kim, "Eedbr: Energy-efficient depth-based routing protocol for underwater wireless sensor networks," in Advanced Computer Science and Information Technology. Springer, 2011, pp. 223-234.

[38] N. Javaid, M. R. Jafri, S. Ahmed, M. Jamil, Z. A. Khan, U. Qasim, and S. S. Al-Saleh, "Delay-sensitive routing schemes for underwater acoustic sensor networks," International Journal of Distributed Sensor Networks, vol. 11, no. 3, p. 532676, 2015.

[39] Y.-S. Chen, T.-Y. Juang, Y.-W. Lin, and I.-C. Tsai, "A low propagation delay multi-path routing protocol for underwater sensor networks," Internet Technology Journal, vol. 11, no. 2, pp. 153-165, 2010.

[40] Z. Guo, G. Colombi, B. Wang, J.-H. Cui, D. Maggiorini, G. P. Rossi et al., "Adaptive routing in underwater delay/disruption tolerant sensor networks," Wireless on Demand Network Systems and Services, pp. 3139, 2008

[41] M. C. Domingo and R. Prior, "A distributed clustering scheme for underwater wireless sensor networks," in Personal, Indoor and Mobile Radio Communications, 2007. PIMRC 2007. IEEE 18th International Symposium on. IEEE, 2007, pp. 1-5.

[42] P. Wang, C. Li, and J. Zheng, "Distributed minimum-cost clustering protocol for underwater sensor networks (uwsns)," in Communications, 2007. ICC'07. IEEE International Conference on. IEEE, 2007, pp. $3510-3515$. 